\title{
Molecular and atomic hydrogen in the environment of hot, massive stars
}

\author{
Nicole St-Louis ${ }^{1}$, S. Gervais ${ }^{1}$, Thierry Morel $^{2}$, and Rene Doyon ${ }^{1}$ \\ ${ }^{1}$ Département de Physique, Université de Montréal, \\ C.P. 6128, Succ. Centre Ville, Montréal (Qc), H3C 3J7, Canada \\ ${ }^{2}$ Imperial College of Science, Technology and Medicine, \\ Blackett Laboratory, Prince Consort Road, London SW7 2BZ, UK
}

\section{Introduction}

Studies of the atomic and molecular components of the interstellar medium in the vicinity of hot, massive stars have the potential of revealing a wealth of information on the way the star affects the distribution and physical state of the surrounding ISM during its various evolutionary phases, as well as potentially providing some information on the star itself and its past evolution.

In this paper we present two examples of studies of the atomic and molecular gas around a massive star. We report on the presence of a large $\mathrm{H}$ i shell around the Wolf-Rayet star WR 134 (HD 191765). This bubble was not blown by the star in its present evolutionary phase but rather while it was an O-type star, on the main sequence. We also report on the discovery of filaments of $\mathrm{H}_{2}$ around a massive star in a much later evolutionary phase, i.e., after it has exploded as a supernova. The $\mathrm{H}_{2}$ filaments we have discovered in the supernova remnant W 44 are probably a direct manifestation of the interaction of the remnant with its surrounding medium.

\section{A large H I shell around the Wolf-Rayet star WR 134}

We have surveyed a $2.6^{\circ} \times 2.6^{\circ}$ field around the WR star WR 134 in the $21 \mathrm{~cm} \mathrm{H} \mathrm{I}$ line and in the 1420 and $408 \mathrm{MHz}$ radio continuum, using the Synthesis Telescope of the Dominion Radio Astrophysical Observatory in Penticton, Canada, in order to search for direct evidence of the interaction between the WR star wind and the ISM.

We found an arc-shaped structure in the $1420 \mathrm{MHz}$ continuum image, which perfectly matches the optical nebula Anon (WR 134). This gas is not visible on our $408 \mathrm{MHz}$ map. This ionized gas could be associated with ejectae from a previous evolutionary phase swept-up by the WR wind and photoionized by the strong UV flux of the star. This suggestion could be confirmed by CNO enrichment measurements in the NW region of the nebula.

Our H I maps show a nearly complete shell at a velocity of $v_{\mathrm{sys}}=-11 \mathrm{~km} \mathrm{~s}^{-1}$ $\left(\mathrm{R}_{\mathrm{s}}=20.9 \mathrm{pc}, v_{\exp }=9.9 \mathrm{~km} \mathrm{~s}^{-1}, M_{\mathrm{H}}=1830 \mathrm{M}_{\odot}\right)$ which, for morphological reasons, we associate with WR 134. The dynamical age of this bubble $\left(1.3 \times 10^{6} \mathrm{yr}\right)$ suggests that it was blown during its main sequence O-star phase. The kinematic distance corresponding to a LSR velocity of $-11 \mathrm{~km} \mathrm{~s}^{-1}$ is about $5 \mathrm{kpc}$, 
which is incompatible with the Cyg OB3 association distance for WR 134 of $2.1 \mathrm{kpc}$. Therefore, we conclude that the $\mathrm{H}$ I bubble was blown in gas already in movement with respect to normal Galactic rotation for this region.

Recent Hipparcos data by Moffat et al. (1998) give a supersonic proper motion for WR $134\left(52 \mathrm{~km} \mathrm{~s}^{-1}\right.$ with $\left.\mathrm{PA}=-38^{\circ}\right)$. This velocity vector points away from the center of our $\mathrm{H}$ I bubble, which strengthens the hypothesis of a physical link with the WR star. The supersonic velocity of the star with respect to the interstellar medium might have generated a bow shock in the SW region, rendering the $\mathrm{H} \alpha$ gas brighter in that direction. A more detailed analysis is presented in St-Louis \& Gervais (1999).

\section{3. $\mathrm{H}_{2}$ filaments in $\mathrm{W} 44$}

The SNR W 44 consists of an elongated filamentary shell which is thought to be directly interacting with its surrounding molecular cloud. Indeed, for six different positions in the remnant, Claussen et al. (1997) have found $\mathrm{OH}$ maser emission, which they suggest are being collisionally excited by $\mathrm{H}_{2}$ molecules in the post-shock gas.

We have obtained narrow 1-0 S(1) $\mathrm{H}_{2}$, [FeII] $1.644 \mu \mathrm{m}, H$ and $K$ images of these six positions using the REDEYE infrared camera on the CFHT in September 1998. The pixel scale of the camera was $\sim 0$. 5 and therefore the $256 \times 256$ pixels of the detector corresponded to a field of approximately $2^{\prime} \times 2^{\prime}$. The data were reduced using the standard procedure including sky subtraction, flat fielding, etc.

We found emission from $\mathrm{H}_{2}$ filaments for all these positions. Conversely, we detect no significant flux from the SNR in our $H$ and $K$ continuum images and therefore we are confident that we are observing genuine line emission in $\mathrm{H}_{2}$. It is tempting to conclude that this $\mathrm{H}_{2}$ gas is shock excited and is responsible for pumping the $\mathrm{OH}$ masers. However, this is inconsistent with our [FeII] $1.644 \mu \mathrm{m}$ observations of these same fields in which we find no significant flux. Indeed, for shocked gas, one would expect a [FeII] $1.644 \mu \mathrm{m} / \mathrm{H}_{2} 1-0 \mathrm{~S}(1)$ ratio much larger than unity (e.g., Graham et al. 1987). Spectra are required in order to estimate line ratios of different $\mathrm{H}_{2}$ transitions and thereby determine whether the molecular hydrogen is shock excited or fluorescent.

\section{References}

Claussen, M.J., Frail, D.A., Goss, W.M., Gaume, R.A. 1997, ApJ 489, 143

Graham, J.R., Wright, G.S., Longmore, A.J. 1987, ApJ 313, 847

Moffat, A.F.J., Marchenko, S.V., Seggewiss, W., van der Hucht, K.A., Schrijver H., Stenholm, B., Lundström, I., Setia Gunawan, D.Y.A., Sutantyo, W., van der Heuvel, E.P.J., De Cuyper, J.-P., Gómez, A.E. 1998, A\&A 331, 949

St-Louis, N., Gervais, S. 1999, AJ submitted 